\title{
Analysis of Bidding Strategy on Decision Support System for Smart Grid
}

\author{
Hongfei Sun \\ School of Economics and Management \\ Northeast Dianli University, NEDU \\ Jilin, China \\ Sunny_bird@126.com
}

\author{
Yanyan Wang \\ School of Economics and Management \\ Northeast Dianli University, NEDU \\ Jilin, China
}

\begin{abstract}
Starting from the basic auction theory in china power market, this paper analyses the influence of electricity cost constitution to bidding strategies, auction theory based on price prediction, auction theory based on the analysis of bidding strategy of opponents, auction theory based on game theory and bidding theory under the contract market deeply, and discusses the corresponding bidding models.
\end{abstract}

Keywords- thermal power plant; electricity price bidding; power generation cost; bidding strategies model

\section{INTRODUCTION}

Our country's power industry is undergoing a marketoriented reform period from the traditional planned economy to the market economy system. Based on what kinds of hypothesis, using what kinds of bidding strategies can power plants get the optimal profits in the competition? How to avoid the risks as much as possible? How to adjust the original bidding ideas and coping strategies? How to actively, selectively participate in the competition, and to make accurate decisions in limited time? All these problems have become a huge challenge that decision makers must face ${ }^{[1]-}$ [3].

\section{POWER Generation Cost Analysis Of THERMAL POWE PLANT}

Power generation cost analysis is the foundation of thermal power plant bidding decision-making. Analyzing the power generation cost constitution, factors effect the change of cost, and how to calculate cost and profit, on the one hand, can lead to internal competition, excavate the potential to reduce cost, grasp the rule cost changes, perfect cost control, and realize the optimal configuration of resources; on the other hand, can provide the reasonable economic basis and wider bidding space for bidding strategies making.

\section{System MARgINAL PRICE ForeCASTING}

For independent power plant, there are three main ways to construct the optimal bidding strategy: forecasting the system marginal price of the next day; estimating the bidding behaviors of other power plants; bidding strategies based on the game theory. There are many methods to forecast marginal price, including probabilistic statistics, time series parameter model approach, grey forecasting method, combination forecast, fuzzy mathematics, artificial neural network method and so on. Here we mainly introduce exponential smoothing prediction method and artificial neural network method. Here mainly introduces exponential smoothing method and artificial neural network method. See Fig.1 and Fig.2

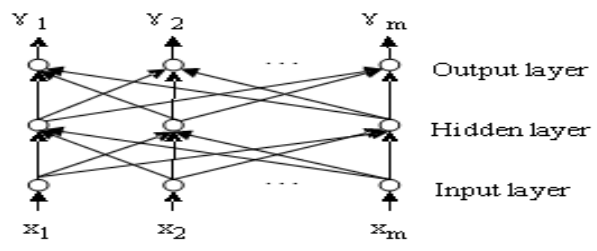

Figure.1. BP Neural network topology structure

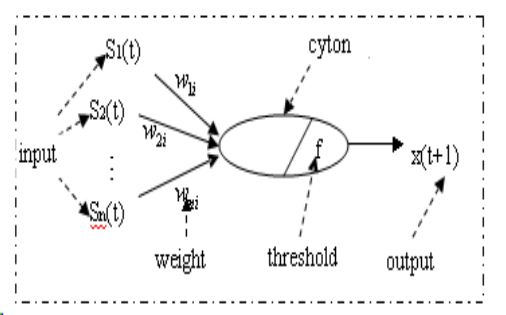

Figure.2. BP Neurons model

\section{Power Plant Bidding Strategies Model}

\section{A. The Bidding Method of Forecasting Price}

In electricity market, to power plant participating in market bidding, their goals are to maximize profit. Therefore, generation bidding is the bidding strategy which will get the maximum returns after determining the reasonable income.

1) Bidding strategy targetfunction

For single unit and single time bidding strategy target function, having said that, power plant aims to maximize generating profit, so the single unit and single time target function is:

$$
\operatorname{Max} \pi(p, q)=p \cdot q-C(q)
$$

To the single unit and more time bidding strategy, because we must consider the continuous operation of units, so based on the single unit and single time, its target function also includes units transfer fee, unit minimum and maximum output, unit ramp rate and other factors. Its target function can be described as follow: 


$$
\operatorname{Max}, \operatorname{MinE}(\pi)=\sum_{t=1}^{T}\left[p_{t} \cdot q_{t}-C_{t}(q)-S_{t}\left(X_{t-1}, u_{t}, u_{t-1}\right)\right]
$$

S.t.

$q_{t(\min )} \leq q_{t} \leq q_{t(\max )}$ unit maximum and minimum output constraints;

$$
\begin{aligned}
& -\Delta q \leq q_{t}-q_{t-1} \leq \Delta q \text { unit ramp rate constraints; } \\
& X_{t+1}=\left\{\begin{array}{ll}
\max \left\{1, X_{t}+1\right\}, & u_{t}=1 \\
\min \left\{-1,-X_{t}-1\right\}, & u_{t}=0
\end{array}\right. \text { unit state transition }
\end{aligned}
$$

equation;

$$
\left\{\begin{array}{l}
u_{t}=1, \quad \text { if }: 1 \leq X_{t} \leq t_{u p} \\
u_{t}=0, \quad \text { if }:-t_{d n} \leq X_{t} \leq-1
\end{array} \quad\right. \text { the maximum }
$$

running time and the smallest outage time of unit $\pi_{t}^{\min } \geq R_{t}$

Among: $S_{t}$ is the start and stop cost of unit, determined by parameter sequence: $X_{t} 、 u_{t} 、 u_{t-1}$. If unit starts from outage state to start-up state from period t- 1 time to t time, so $S_{t}$ presents start-up cost; if this unit is in operation state all the time, $S_{t}$ is 0 ; if unit starts from start-up state to outage state, $S_{t}$ represents that outage cost consists of shut-down cost $C_{d o w n}$ and shut-down fixed cost $C_{f}$, namely, $S_{t}=C_{f}+C_{d o w n}$, and at this time target function becomes minimum downtime costs, namely $\operatorname{Max} E(\pi)->\operatorname{MinE}\left(S_{t}\right) ; u$ represents unit control status, 1 for boot and 0 for shutdown. $X_{t}$ represents the unit state in $t$ time, when units put into operation $X_{\mathrm{t}}>1$, and when unit allows outage $X_{t}<0 ; R_{t}$ is the minimum income when unit offset its variable cost in time $t$.

2) Strategies selection of the forecast price bidding method

The optimal point of units quotations: by forecasting we can get price random variable mean value $p_{t}$, standard deviation $\sigma_{p t}$, which can be substituted into the objective function to get the optimal capacity in time $t: Q_{t}{ }^{*}=(p$ $b) /(2 a)$, and then determine the unit optimal quotation point $\left(p_{t}, q_{t}^{*}\right)$ in time $t$.

The economic constraint points of bidding unit: to the minimum economic capacity constrained by unit economic $q_{t}^{\min }=\sqrt{c / a}$, its corresponding price is:

$$
p_{t}^{\min }=2 \sqrt{a \cdot c}+b
$$

Based on the above analysis and combining with the minimum and maximum output of unit, the unit quotation power range can be determined:

$$
\left[\max \left\{q_{\min }, q_{t}^{\min }\right\}, \min \left\{q_{\max }, q_{t}^{*}\right\}\right]
$$

3) Single time unit quotation scheme

After determining the unit feasible quotation power range by the above analysis, units can find several bidding scopes $\left(p_{t}, q_{t}\right)$ from the above range in time $t$, determine and submit bidding strategies to organization in meeting the rule that the bidding curve must be non-decreasing claimed by the market. Here conversion coefficient method is adopted to determine and declare energy price: introduce a conversion coefficients and make:

$$
\rho_{t}=\bar{p}_{t} \times m, \quad m \subset\left[m^{*}, 1\right], \text { among } m^{*}=p_{t}^{\min } / \bar{p}_{t}
$$

The choice of $\mathrm{m}$ generally does not exceed 1 , because 1 corresponds to the average settlement price predicted in time $t$. Obviously, declaring this price is not good for bidding. Now, between the bidding power range $\left[q_{\text {toplimit }}\right.$, $\left.q_{\text {lower limit }}\right]$ and the range of $m$, using different equal segments or following certain requirements to get the value of $m$ and gain several bidding quotation $p_{t}$. According to the optimal power corresponded by the maximum profit function, we know: $Q_{t}=\left(p_{t}-b\right) / 2 a$, which is the corresponding quotation electricity.

\section{B. The Bidding Method of Forecasting Rivals}

The bidding method of forecasting rivals is to seek the optimal bidding strategy on the basis of forecasting the bidding energy price of rivals known and system demand for electricity load forecasting.

1) Condition assumptions

The decision-making behavior of bidders is rational, namely bidders will choose the optimal bidding strategies according to the bidding information obtained and pursue long-term expected profit maximization and the short-term or eyes profit maximization. At the same time, spare, auxiliary service will not be considered and assume that there is not joint bidding among power plants. And before bidding, power plant has known the power load forecasting demand of each period, and learned the power and price of the opponents by forecasting.

\section{2) The optimal bidding strategies model}

Here mainly take the bidding strategy of single period, single unit and two power suppliers participating in bidding as the example, power plants quote price in five-point method, quotation form is a step-like increasing bidding curve. Suppose that the bidding strategy form of bidding power plant $j$ is obtained by forecasting, as described below, bidding form simplifies into integer form, so according to opponent quotation, we can determine the optimal bidding through the following methods. Suppose the bidding form of opponent $\mathrm{j}$ is:

Given power: $\Phi 1, \varphi 2, \ldots, \varphi j, \ldots, \varphi 5(m w)$

Generation bidding: $P_{1}, \rho_{2}, \ldots, \rho_{j}, \ldots, \rho_{5}(Y / m w)\left(\rho_{j}+1>\rho_{j}\right)$

Type, command $\alpha=\varphi_{j}+1-\varphi_{j}=\left(\varphi_{5}-\varphi_{1}\right) / 4$, namely $\varphi_{2}=\varphi_{1}+\alpha, \varphi_{3}=\varphi_{1}+2 \alpha, \varphi_{4}=\varphi_{1}+3 \alpha, \varphi_{5}=\varphi_{1}+4 \alpha$, the bidding form of power plant itself is:

Given power: $H_{1}, h_{2}, \ldots, h_{i}, \ldots, h_{5}(m w)$

Generation power: $P_{1}, p_{2}, \ldots, p_{i}, \ldots, p_{5}(Y / m w)\left(p_{j}+1>p_{j}\right)$

Type, command $\alpha=h_{j}+1-h_{j}=\left(h_{5}-h_{1}\right) / 4$, namely $h_{2}=h 1+\alpha, h_{3}=h_{1}+2 \alpha, h_{4}=h_{1}+3 \alpha, h_{5}=h_{1}+4 \alpha$ 
Apparently, the bidding aim of power plant $i$ is to find the bidding strategy targeting at opponent, and to obtain the maximal profit, which need to meet the below equation:

$$
\operatorname{Max}\left(\pi_{i}\right)=\left[p_{i} \cdot H_{i}-C_{i}\left(H_{i}\right)\right] \cdot V_{i} \quad i=1,2,3,4,5
$$

Type, $c_{i}\left(h_{i}\right)=a h_{i}+b, a>0, b \geq 0 ; v_{i}$ represents winning a bid or not, if winning its value is 1 , not is 0 , and its value is determined by the following formula:

$V_{i}=1$ if $\left[\left(h_{i}+\varphi_{j-1}<d \leq h_{i}+\varphi_{j}<h_{i}+\varphi_{j}+1\right)\right.$ and $\left(p i=\rho_{j+1}-1\right.$; $\left.\left.p_{i+1}=p_{i}+1 ; p_{i-1}=p_{i}-1\right)\right]$

$V_{i}=0 \quad$ otherwise

S.t. $H_{1}=\max \left(h_{\min }, h_{\min }\right) ; h_{5}=\min \left(h_{\max }, h^{*}\right)$

$H_{\min }, h_{\max }$ is the minimum and maximum output of unit; $h_{\min }, h^{*}$ is the minimum economic output and the best output in this period. All these conditions explain that given power $i$ will win the bid if the quotation $p_{i}$ given power $H i$ corresponding to is less than the generation power $\rho_{j+1}$ of power plant $j$, and at this time the sum of $\varphi_{j}$ and $H i$ can satisfy the control condition when $v_{i}=1$. Of course in this time if the value of $p_{i}$ is $\left(\rho_{j+1}-1\right)$, its net profit will reaches the maximum in this form.

\section{3) Uncertainty and randomness analysis}

Because the main role of models is to predict results to the bidding strategies of opponent, which includes multiple prediction and the corresponding probability, so to power plant $i$, against each group prediction bidding combination, determine first the corresponding bidding strategies, at the same time in the bidding strategies, and then determine the optimal bidding strategies based on the prediction probability. To this can use the Monte Carlo method to solve.

\section{Game Theory Bidding Strategy}

Different market environments determine the different premise of game model, frequently, the results are different. Directing at these mainly factors and the current facts in power market of our country, puts forward the below bidding game hypothesis:

- Power market adopts the bilateral trade form rather than joint venture form, and uniform market-clearing price system;

- Power plant is the intermediary institution between thermal power plant and companies who purchase power;

- The nature of the goods belongs to before market or real-time balance market;

- Suppose there is no network congestion or transport bottlenecks.

1) Non-cooperative game model of bidding strategies

Based on the above game assumptions, by analyzing the power plant generation bidding, before bidding power plant can gain corresponding information, assumptions are as follows:

Settlement price in trading mainly is the function of total output of all power plants who participate in trading, and is the kind of drop demand function, command: $D=a$ $b \times p=\sum q i(p), \quad p$ presents system marginal price;

Regional grid has $n$ power plants, which bid away at the same period, while during this time they don't know the bidding of other power plants;

Generation cost of power plants are all second cost function form, and unknown to the specific of other plants, the cost function of the $i$ power plant is: $C_{i}\left[q_{i}(p)\right]=0.5 a_{i} q_{i}^{2}(p)+b_{i} q_{i}(p)+d_{i}, \quad i=1,2, \ldots, n$;

In certain period of time in the future, bidding strategies of each power plant in spot market is described by each supply function, for: $Q_{i}(p)=\alpha_{i}+\beta_{i} \times p \quad i=1,2, \ldots, n$, among $\beta_{i}>0$;

Generation company in the cash market has no collusion behavior with each other;

Only discuss single time bidding strategies;

From the above assumptions, we know, this noncooperative game is the static type of incomplete information game, namely, Bayesian Game;

2) Bidding strategies for solving

Based on the above assumptions, power plant aims to maximize the profit, profit function is: $\operatorname{Max}_{i}=p \times q_{i}(p)$ $c_{i}\left[q_{i}(p)\right]$, thus, bidding strategies of power plant $i$ is:

$$
\left\{\begin{array}{r}
\alpha_{i}=\frac{-b_{i}\left(b+\sum_{j=1, j \neq i}^{n} \beta_{j}\right)}{1+a_{i}\left(b+\sum_{j=1, j \neq i}^{n} \beta_{j}\right)} \\
\beta=\frac{b+\sum_{j=1, j \neq i}^{n} \beta_{j}}{1+a_{i}\left(b+\sum_{j=1, j \neq i}^{n} \beta_{j}\right)} \\
p=\frac{A-\sum_{i=1}^{n} \alpha_{i}}{b+\sum_{i=1}^{n} \beta_{i}} \\
q_{i}=\frac{\left(p-b_{i}\right) \cdot\left(b+\sum_{j=1, j \neq i}^{n} \beta_{j}\right)}{1+a_{i}\left(b+\sum_{j=1, j \neq i}^{n} \beta_{j}\right)}
\end{array}\right.
$$

To prove it, the company balanced supply function obtained from the above is the maximum point of overall situation, and after determining the system settlement price and the opponent supply function and substituted into 4-4 the third, then can get the balanced power generation $q_{i}$ of power suppliers.

\section{Bidding Models Based on Price-Difference Contract}

1) Model assumptions

To bidding strategies based on the follow assumptions:

- $\quad$ Considering a power market existing $n$ power plants, load demand function of a future time period is: $D(p)=a-b p$, among $p$ is spot market price in this time, $a$ and $b$ are constants greater than 0 ;

- Every power plant has second cost function: 


$$
C_{i}\left(q_{i}\right)=0.5 c_{i} q_{i} 2
$$

Among $q_{i}$ is the production yields of the $i$ power plant in this time, $c_{i}$ is constant greater than 0 . Power plant has linear marginal cost which equals $c_{i} q_{i}$;

- In spot market of future time, bidding strategy of each power plant is described by each supply function, which is:

$$
Q_{i}(p)=\alpha_{i}+\beta_{i} \times p \quad i=1,2, \ldots, n, \quad \beta i>0
$$

- Power plant can sign a two-way spread contract with power supply companies or users for power selling in the further, the price decided in contract is described in $f(f \geq 0)$, electricity contract of power plant $i$ is $x_{i}$. According to the definition of pricedifference contract, the expenses power plant $i$ will expend (or receive, if is negative) in the future time is $(p-f) x_{i}$.

- In price-difference contract market, the decrease of electricity contract of the $i$ power plant will cause the increase of other power plants, and the increasing degree can be described in conjectural variation $\partial x_{j} / \partial x_{i}(j=1,2, \ldots, n, j \neq i)$ ( when $\mathrm{n}=2$, conjectural variation is $\left.d x_{2} / d x_{1}\right)$, meet:

$$
\left\{\begin{array}{l}
\frac{\partial x_{j}}{\partial x_{i}} \in[-1,0] \quad \forall j \neq i \\
\sum_{j=1, j \neq i}^{n}\left(\frac{\partial x_{j}}{\partial x_{i}}\right) \in[-1,0]
\end{array} \quad i=1,2, \cdots, n\right.
$$

- None of power plants has collusion behaviors in spot market and contract market.

2) The spot market model

The equilibrium price $p$ of power spot market is determined through the market clearing, namely:

$$
A-b p=\sum_{i=1}^{n} q_{i}(p)
$$

The aim of every power plant is to make profit maximization, among, the profit $R_{i}$ of power plant $i$ in discussing time equals its income in the spot plus the net income of price-difference contract, and minus generation cost, namely:

$$
R_{i}=p q_{i}(p)+(f-p) x_{i}-C_{i}\left(q_{i}(p)\right)
$$

Among: $X_{i}$ is electricity contract.

When the power profit achieves the maximum, reciprocal that profit function corresponds to is 0 , by $4-9$, we know supply function meets differential equation:

$$
q_{i}(p)=x_{i}+\left(p-c_{i} q_{i}(p)\right)\left(b+\sum_{j=1, j+i}^{n} \frac{d q_{j}}{d p}\right)
$$

Simultaneity formulas above from 4-5 to $4-10$, we can obtain the spot market price $p$ and the generated energy of balanced supply function for the $i$ power plant.

$$
\begin{gathered}
p=\frac{1}{b+\sum_{j=1}^{n} \beta_{j}}\left[A-\sum_{j=1}^{n} \frac{\beta_{j} x_{j}}{b+\sum_{k=1, k \neq j}^{n} \beta_{k}}\right] \\
q_{i}=\frac{\beta_{i}}{b+\sum_{j=1}^{n} \beta_{j}}\left[A-\sum_{j=1, j \neq i}^{n} \frac{\beta_{j} x_{j}}{b+\sum_{k=1, k \neq j}^{n} \beta_{k}}+x_{i}\right]
\end{gathered}
$$

\section{3) Contract market model}

Suppose enough buyers in contract market are risk neutral, have rational expectation, so contract price $f$ will equal expected spot price. From type 4-11, contract price will depends on the electricity contract of each power plant. Because contract price equals expected spot price, the profit of power plant can be written as:

$$
R_{i}=f\left(x_{1}, x_{2}, \cdots ; x_{n}\right) q_{i}\left(x_{1}, x_{2}, \cdots ; x_{n}\right)-\frac{1}{2} c_{i} q_{i}\left(x_{1}, x_{2}, \cdots ; x_{n}\right)^{2}
$$

When profit is the optimal, first derivative of profit to electricity contract is 0 , namely: $D r_{i} / d x_{i}=0$

Combining the above type (5) (12), we know the electricity contract of the $i$ power plant meets:

$$
\begin{gathered}
x_{i}=-q_{i} \frac{\left(\sum_{k=1}^{n} \beta_{k}+b\right) W_{i}}{\beta_{i}\left(1-W_{i}\right)} \\
W i=\sum_{j=1, j \neq i}^{n}\left(1-c_{j} \beta_{j}\right) \frac{\partial x_{j}}{\partial x_{i}}
\end{gathered}
$$

By simulating this model and further analyzing, we know: the bigger the absolute value of conjectural variation $\partial x_{i} / \partial x_{j}$, the bigger the electricity contract $x_{i}$ and generation power $q_{i}$ of power plant $i$. The increased speed of $x_{i}$ greater than $q_{i}$ means the more enthusiasm for power plant $i$ entering contract market.

\section{CONCLUSIONS}

Bidding decision-making is an unstructured process, price of bidding strategies is always based on various forecasting model. However, the uncertainty of model determines that quotation exist some risks. So, if modern intelligent decision-making techniques could be used to help bidding, and make full use of the advantages of computer in information processing and managing ${ }^{[4]}$, the 
efficiency will be greatly improved. For now, domestic and foreign scholars have researched and widely discussed the bidding strategies theory of power plant, and some relevant auxiliary support system model also have been put forward by some researchers, but, making use of intelligent decision support technology to assist power plant bidding is rare, which is also the focus of our research in the future ${ }^{[5]}$.

\section{REFERENCES}

[1] Shangfei Xiong and Xiaoyan Zou, The forecast review of price risk and volatility of electricity market value, Power System Protection and Control, 2014(2)

[2] Zhigang Wang, The pricing strategy of Power Marketing, Hua Zhang, 2013 (2)

[3] Wei Zheng, The role of electricity in power marketing and marketing services of electricity, Technological Development of Enterprise, 2013(21)

[4] Xiangting Chen and Xiaofeng Lai, Taker bilateral bidding strategy based on fuzzy neural network, Value Engineering, 2013 (11)

[5] Qiang Han and Yan Liu, The impact analysis of information disclosure on bidding strategy, Energy Technology and Economics, 2012(07) 